\title{
Massive stellar populations in the infrared
}

\author{
Claus Leitherer \\ Space Telescope Science Institute, \\ 3700 San Martin Drive, Baltimore, MD 21218, USA
}

\begin{abstract}
An overview of some key results related to hot, massive stars and starbursts in the infrared is given. The wavelength range covers the groundand space-based near-infrared, the mid- and far-IR as probed by several space missions, and the sub-millimeter regime. I discuss how these observations permit an unbiased view of dust-obscured stars and how they are related to data at optical and ultraviolet wavelengths.
\end{abstract}

\section{Introduction}

Observational studies of massive stars have traditionally been biased toward the optical/ultraviolet, where the most significant spectral lines are and where the stellar energy distribution peaks. This is also the domain where hot stars dominate over their cooler descendants in a stellar population. Quite ironically, the optical-UV can be the least favorable wavelength region for observations of hot, massive stars in starburst galaxies. Stars, gas, and dust are co-spatial, and the most intense star-formation sites are often deeply obscured by dust. Only observations at sufficiently long wavelengths can unveil the underlying stars, either directly, or via the radiation from surrounding gas and dust. I will address a handful of topics, arranged by wavelength domain from the near-infrared to sub-millimeters, highlighting the impact of IR data on our understanding of massive stellar populations. The subjects cover the determination of starburst masses, the IMF of massive stars, the heating of the interstellar medium (ISM), the connection between starbursts and AGN, and the global energy budget of starbursts.

\section{Red-supergiant features and starburst masses}

Determining the stellar mass in a starburst and/or the low-mass end of the initial mass function (IMF) is notoriously challenging. Massive stars are overwhelmingly luminous for a typical population mix, precluding any direct detection of the light from low-mass stars. Yet, it is the low-mass population where most of the stellar mass is concentrated and whose mass fraction determines the starformation efficiency.

The most direct technique to obtain the starburst mass relies on gravity, not on radiation. Velocity dispersion measurements of the gas in the center of M 82 provide strong support for a stellar population which is overluminous for its mass, and therefore for a top-heavy IMF (Rieke et al. 1993). Stellar 
velocity dispersions of starburst regions have remained elusive until very recently. In a pioneering study, Ho \& Filippenko (1996a,b) detected and resolved faint absorption features from red supergiants in optical spectra of the super star clusters in NGC 1569 and NGC 1705. This technique has been applied to clusters in other starburst galaxies, such as M 82 (Smith \& Gallagher (2001). RSGs are the only species in a young stellar population whose spectral lines are detectable and sufficiently narrow to provide the velocity dispersion, whose value is of order $10 \mathrm{~km} \mathrm{~s}^{-1}$ for a $10^{6} \mathrm{M}_{\odot}$ cluster.

Observations of RSG features in the near-IR have two distinct advantages over the optical: $(i)$ an approximately ten-fold decrease of dust attenuation; and (ii) an almost complete absence of dilution from stars not in the RSG phase. The most prominent features in the $H$ - and $K$-bands are the first and second overtones of $\mathrm{CO}$ at $2.29 \mu \mathrm{m}$ (2-0 band head) and $1.62 \mu \mathrm{m}(6-3$ band head) and the $\mathrm{Si}$ I absorption at $1.59 \mu \mathrm{m}$ (Oliva et al. 1995).

Böker et al. (1999) observed the nuclear stellar cluster in the nearby spiral galaxy IC 342. Their high-resolution $(R=20000)$ spectra of the CO band head at $2.29 \mu \mathrm{m}$ allowed them to derive a line-of-sight stellar velocity dispersion of $33 \mathrm{~km} \mathrm{~s}^{-1}$. Under the assumption of an isotropic velocity distribution, a massto-light ratio in the $K$-band of $M / L_{K}=0.05$ and a cluster mass near $10^{7} \mathrm{M}_{\odot}$ are found. Comparison of the derived mass-to-light ratio with stellar population synthesis models suggests an age around $10^{7} \mathrm{yr}$.

$H$-band echelle observations of the super star clusters A and B in NGC 1569, together with appropriate RSG template spectra were obtained by Gilbert \& Graham (2002). The data have a resolution and S/N comparable to those of the optical spectra analyzed by Ho \& Filippenko (1996a; see above), but are superior in two ways: the spatial information allows the separation of the two cluster components in NGC 1569-A (Maoz et al. 2001), and the longer wavelength minimizes the contamination from non-RSG stars. Cluster B has a velocity dispersion of $7.5 \pm 0.2 \mathrm{~km} \mathrm{~s}^{-1}$, which implies a dynamic mass of $2.3 \times 10^{5} \mathrm{M}_{\odot}$ from the virial relation. The cross-correlation function of cluster $\mathrm{A}$ is asymmetric and can be fit by two Gaussian components separated by $21.4 \mathrm{~km} \mathrm{~s}^{-1}$, each with a velocity dispersion of $10.4 \pm 0.4 \mathrm{~km} \mathrm{~s}^{-1}$. The implied dynamic masses for the two components are $3.9 \times 10^{5}$ and $4.4 \times 10^{5} \mathrm{M}_{\odot}$. This may be compared with a total mass of cluster $A$ of about $10^{6}$ derived by Ho \& Filippenko under the assumption of a single component. The studied clusters have a normal IMF, with no evidence for a low-mass truncation from the dynamic mass determination.

Near-IR velocity dispersions, both from absorption and emission lines, are still around the instrumentation limits. Rapid technological progress will make this technique feasible for even less massive and more distant starburst clusters.

\section{Hot, massive stars in dusty starbursts}

In contrast to RSGs, hot stars have no sufficiently strong features that would be detectable in a population longward of $\sim 1 \mu \mathrm{m}$. However, the mid-IR includes numerous nebular fine-structure lines with ionization energies of tens of $\mathrm{eV}$. These lines are suitable tracers for the shape of the stellar ionizing spectrum, and therefore for the properties of the upper end of the IMF.

Thornley et al. (2000) carried out an ISO spectroscopic survey of 27 starburst galaxies with a range of luminosities from $10^{8}$ to $10^{12} \mathrm{~L}_{\odot}$. [Ne III] $15.6 \mu \mathrm{m}$ 

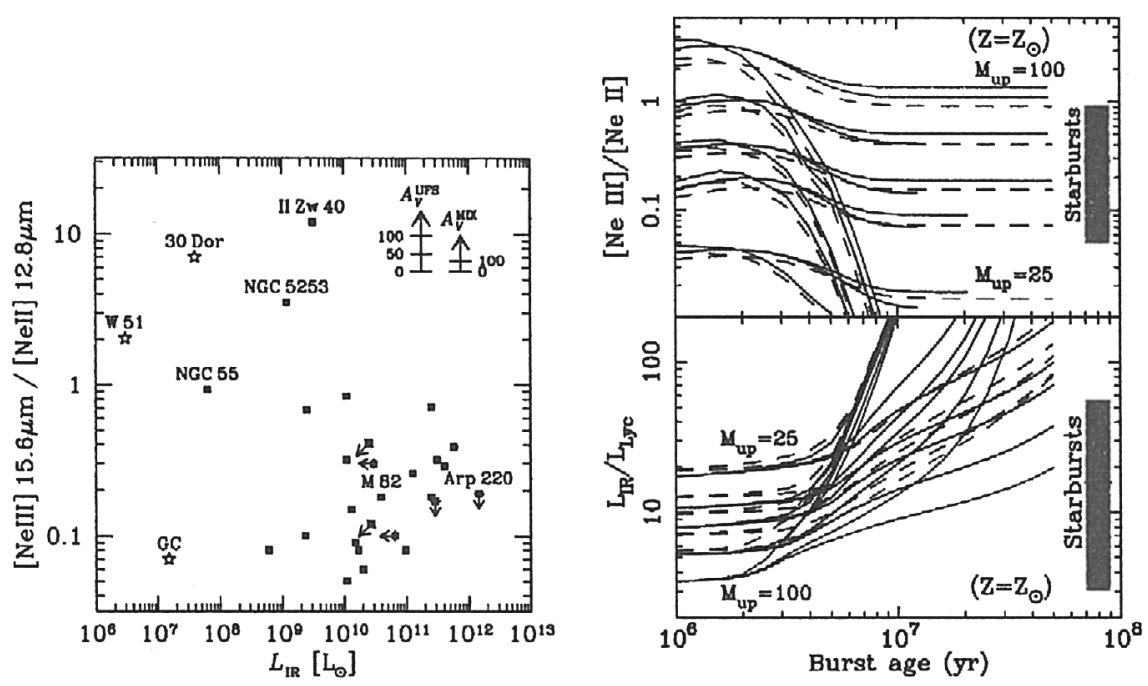

Figure 1. Left: Ratio of [Ne III] $15.6 \mu \mathrm{m}$ over [Ne II] $12.8 \mu \mathrm{m} v s . L_{\mathrm{IR}}$ for a sample of starburst galaxies and nearby star formation regions measured with ISO-Sws. Arrows in the upper right indicate the effects of dust attenuation. Right: [Ne III]/[Ne II] ratios (upper) and $L_{\mathrm{IR}} / L_{\text {Lyc }}$ line ratios (lower) for starburst models with $M_{\text {up }}$ between 25 and $100 \mathrm{M}_{\odot}$. For each $M_{\text {up }}$, three burst models are shown for timescales of 1,5 , and $20 \mathrm{Myr}$. The vertical bar on the right side indicates the range of measured ratios. From Thornley et al. (2000).

and [Ne II] $12.8 \mu \mathrm{m}$ lines are particularly useful. The ionization potentials of neutral and ionized $\mathrm{Ne}$ are 22 and $41 \mathrm{eV}$, respectively, the two lines are very close in wavelength $(12.8$ and $15.6 \mu \mathrm{m})$, and they have similar critical densities. This makes the line ratio a sensitive probe for various star formation parameters, in particular the upper mass cutoff of the IMF $\left(M_{\mathrm{up}}\right)$ and the age and duration of the starburst. The result of the ISO survey is in Figure 1 (left). The neon ratios of the sample objects are generally found to be between 0.05 and 1.0; however, the metal-poor starburst galaxies NGC 5253 and II Zw 40 have neon ratios of 3.5 and 12, respectively. It is instructive to compare these neon ratios to observations of nearby high-mass star-formation sites, such as W 51 , 30 Doradus, and the central parsec of the Galaxy. The neon ratios for W 51 and 30 Doradus are higher than for the starburst galaxy sample, while that for the Galactic center is lower. The ISO-sws data thus generally confirm that powerful dusty starbursts exhibit low nebular excitation (e.g., Doherty et al. 1994). The average [Ne III]/[Ne II] ratio in starburst galaxies is a factor of 2 to 3 lower than in individual Galactic compact $\mathrm{H}$ II regions.

Thornley et al. (2000) modeled the H II regions as ionization-bounded gas clouds photoionized by central evolving star clusters. The computed Ne ratios (for a Salpeter IMF with different values for $M_{\mathrm{up}}$ ) as a function of burst age and duration are shown in Figure 1 (right). The important result is that on average stars more massive than about 35 to $40 \mathrm{M}_{\odot}$ are deficient in the observed sample. Either they never formed because of a peculiar IMF, or they have already disappeared as a result of aging effects. 
An upper-mass cutoff as low as $40 \mathrm{M}_{\odot}$ is difficult to reconcile with the ubiquitous evidence of very massive stars with masses of up to $100 \mathrm{M}_{\odot}$ in many starburst regions (e.g., Leitherer et al. 1996; Massey \& Hunter 1998; González Delgado et al. 2002). Therefore the alternative explanation, an aged starburst seems more plausible. Under this assumption, stars of masses $50-100 \mathrm{M}_{\odot}$ are initially formed in most galaxies, but the starbursts are observed at an epoch when these stars are no longer present. This implies that the inferred burst durations must be less than a few Myr (Figure 1). Such short burst timescales are surprising, in particular for luminous, starburst galaxies whose the dynamical timescales can exceed tens of Myr. However, supernovae and stellar winds can induce large negative feedback. Starburst models (Leitherer et al. 1999; Thornley et al. 2000) as well as observations of galactic superwinds (Heckman et al. 1990) indicate that $1 \%$ to $2 \%$ of the bolometric luminosity of starbursts is converted into mechanical energy in superwinds. This mechanical energy input could remove interstellar gas from the star-formation site on a time scale much shorter than the gas consumption timescale, which can be hundreds of Myr.

Both the peculiar IMF or the short starburst timescales in dusty, IR-bright starbursts are quite unexpected and pose a challenge to conventional models in which the starburst is fed by gas inflow to the nucleus over tens of Myr as a result of angular momentum loss. Clearly, additional observational verification is desired since this interpretation rests on the assumption that the soft radiation field results from a deficit of massive stars. A case in point is the UV-optical study of NGC 3049 by González Delgado et al. (2002). This metal-rich starburst galaxy exhibits an emission-line spectrum of relatively low excitation, whereas the UV stellar-wind lines clearly prove the presence of massive, ionizing stars following a normal Salpeter-type IMF. Indirect star-formation tracers, like nebular lines, still require careful calibration, in particular when applied to dusty, metal-rich starbursts.

\section{The heating mechanism of the interstellar medium}

The stellar ionizing thermal luminosity decreases with time since the overall evolutionary trend is towards lower stellar temperature. Therefore the relative proportion of the non-thermal (i.e., energy input from winds and supernovae) and thermal luminosities increases with time, with the two quantities being comparable in an instantaneous starburst of age $10 \mathrm{Myr}$. After that epoch, the powering of the interstellar environment becomes almost totally determined by the non-thermal energy input. Thus, the emission-line characteristics of the ionized gas change from a photoionized to a shock-ionized spectrum.

The high-ionization species of [S IV] $10.5 \mu \mathrm{m}$ and [O IV] $25.9 \mu \mathrm{m}$ are of special interest for constraining the presence of very hot stars $\left(T_{\text {eff }} \approx 50000 \mathrm{~K}\right)$ and shock-ionized gas in the star forming regions. The [O IV] line is a powerful diagnostic because it is collisionally de-excited in stellar winds and hence serves as a purely nebular hardness indicator for photons with energies above $55 \mathrm{eV}$.

In the sample of [O IV] emitting starbursts of Lutz et al. (1998), II Zw 40 and NGC 5253 show the strongest excitation (measured by $[\mathrm{Ne} \mathrm{III}] /[\mathrm{Ne} \mathrm{II}]$ ) and the largest [O IV] strength, and stand out in their nebular He II 4686 emission, indicative of high excitation. A significant number of Wolf-Rayet (WR) stars has been detected in these galaxies (Vacca \& Conti 1992; Schaerer et al. 1999). 
The optical detection of photons above $54 \mathrm{eV}$ and their spatial association with the mid-IR [O IV] line suggests a direct link between the nebular He II and [O IV] emission. The optical He II 4686 has been used before to model the WR content in starburst galaxies (e.g., Schaerer \& Vacca 1998). Its usefulness is compromised by dust obscuration effects and by its dual formation mechanism: as a resonance line, it can form in the ISM and in the winds of the ionizing stars themselves. Although the latter contribution has a much broader profile, typical spectra often have insufficient quality to separate the two components. The mid-IR [O IV] line traces the same energy regime as the He II line $(>54 \mathrm{eV})$ but does not form in winds and is free of dust obscuration effects.

Previous photoionization models indicate a short phase in the evolution of a stellar population with sufficient photons above $54.4 \mathrm{eV}$ to naturally produce the He II, [O IV], or [S IV] lines at levels comparable to the observations. The emission is due to the presence of hot WR stars at ages 3-4 Myr (Schaerer \& Stasińska 1999). However, these older models rely on the first generation of expanding WR atmospheres calculated by Schmutz et al. (1992), which are suspected to produce overly hard radiation fields (Bresolin \& Kennicutt 2002). Models with the new generation of blanketed atmospheres discussed by Smith et al. (2002) may lead to some revision of these results. Nevertheless, it is commonly accepted that hot stars in unevolved starbursts are at least partially responsible for the [O IV] and related lines.

Alternatively, the observed relation between [O IV] and [Ne III] / $\mathrm{Ne} \mathrm{II]} \mathrm{is} \mathrm{well}$ reproduced by models accounting consistently for both shock and photoionization effects occurring in starburst regions. The shock component becomes increasingly more important in older starbursts, such as M 82 (Viegas et al. 1999), as expected from the higher non-thermal energy input from supernovae. The critical parameters which reproduce the observed trend of the mid-IR line ratios are the pre-shock density and the shock velocity. Most of the shocks are produced in low-density and low-velocity clouds, which represent the bulk of the ionized gas in starburst galaxies. The difference between young ( $\mathrm{H}$ II galaxies) and older starbursts (M 82-like) can be interpreted in terms of an evolutionary sequence. In the former, the starburst is too young to release large amounts of mechanical energy through supernova explosions whereas in the latter the kinetic energy competes with the ionizing radiation in the heating of the ISM.

\section{The starburst-AGN connection}

The central accretion rate in the prototypical Seyfert2 galaxy NGC 1068 is about $0.1 \mathrm{M}_{\odot} \mathrm{yr}^{-1}$ (Veilleux 2001), close to the star-formation rate in M 82. Therefore it is natural to suggest a common feeding mechanism for both a starburst and an AGN, i.e., infall of gas to the galaxy center. If so, starbursts and AGN should coexist and exhibit a close connection.

Hot stars are a significant component of the UV light in many bright Type 2 AGN. The stars in these AGN are typically obscured and reddened by dust, with attenuation factors of $5-10$ at $1500 \AA$. The implied UV luminosities of the starbursts range from $10^{8-9} \mathrm{~L}_{\odot}$ in low-luminosity AGN and $10^{10-11} \mathrm{~L}_{\odot}$ in Seyfert galaxies. Massive stars are clearly energetically significant in AGN. However, stars have been directly detected in relatively few cases, and these are biased in favor of galaxies with high UV surface brightness. Cid Fernandes et al. (2001), 
applying detailed semi-empirical population synthesis models, find about $40 \%$ of Seyfert 2 galaxies to have composite starburst+AGN spectra.

Analysis of stellar features is preferable over nebular diagnostics, which can often be degenerate. Nevertheless, since the nebular emission lines are by far the strongest features in an AGN spectrum, numerous efforts have been made to utilize them as a tracer of the starburst population. The fundamental challenge is to disentangle between extreme-UV radiation field of stars vs. the power-law spectrum emitted by the gas accreting onto a black hole. Often, both types of ionizing spectra can produce very similar optical and IR emission lines, and a carefully fine-tuned starburst spectrum can often mimic the same nebular emission-line spectrum as does a power law spectrum from an AGN.

An important result of the ISO mission was a set of mid-IR diagnostics which can break the degeneracy. The differences between the spectra of starbursts and AGN in the mid-IR are striking. Starbursts have strong low-excitation fine-structure lines, few and/or weak high-excitation lines, little dust emission below $10 \mu \mathrm{m}$, and very strong polycyclic aromatic hydrocarbon (PAH) bands. In contrast, Seyfert 2 galaxies have fainter emission lines, but with much higher excitation (e.g., the much stronger [O IV] $25.9 \mu \mathrm{m}$ line). The dust continuum is much stronger in the AGN-type spectra as well. These trends hold for even more powerful AGN up to the highest observed luminosities (Genzel \& Cesarsky 2000).

Diagnostic diagrams are powerful tools for an empirical characterization of the excitation state of a source. Genzel et al. (1998) introduced the [O IV] $25.9 \mu \mathrm{m}$ to [Ne II] $12.8 \mu \mathrm{m}$ line flux ratio versus the strength of the PAH-bands to discriminate between starbursts and AGN. This ISO-based diagnostic diagram clearly separates known star forming galaxies from AGN. The diagram is insensitive to dust extinction if the emission lines and the bands are affected by a similar amount of extinction. [O IV], although weak, is often detected in starburst galaxies. As discussed before, its origin is due to a combination of fast, ionizing shocks powered by supernovae and stellar winds and of the hard UV radiation from hot Wolf-Rayet stars.

The mid-IR diagnostic diagram is most powerful when applied to dusty, IR-luminous galaxies, where the nature of the underlying source is not known a priori. Ultra-luminous IR galaxies (ULIRGs) are of particular interest, as the starburst versus AGN debate is still ongoing in these objects. When studied with this method, starbursts dominate up to luminosities of about $2 \times 10^{12} \mathrm{~L}_{\odot}$, above which AGN become energetically more and more important. The most luminous starbursts are detected in galaxies with luminosities close to $\sim 10^{13} \mathrm{~L}_{\odot}$. This limit corresponds to the maximum star formation rate of $\sim 10^{3} \mathrm{M}_{\odot} \mathrm{yr}^{-1}$ of a gas-rich spheroid undergoing a monolithic collapse on a dynamical timescale.

There is now ubiquitous evidence for starbursts in IR-luminous AGN over several decades in luminosity. While the precise evolutionary scheme is still unknown, it seems likely that the gas supply feeding the central AGN is capable of sustaining the circumnuclear starburst as well.

\section{The energy budget of dusty starbursts}

Energy conservation dictates that the attenuated light in the UV must be reemitted in a different waveband. The dust grains scattering and absorbing the 
stellar light have typical sizes of about $0.1 \mu \mathrm{m}$ and equilibrium temperatures of tens of $\mathrm{K}$. The corresponding emission peaks in the far-IR around $60 \mu \mathrm{m}$. Therefore, the net effect of dust obscuration is a redistribution of the stellar UV-optical flux to the far-IR. In the local universe, about $80 \%$ of the stellar UV photons are hidden from direct view and can only be recovered as reprocessed light in the far-IR (Calzetti 2001). The starburst energy distribution always peaks around $60 \mu \mathrm{m}$, irrespective of the amount of reddening. This property has important consequences for the prospects of detecting starburst galaxies at high redshift(see below).

If dust acts like a foreground screen, we expect a simple scaling between the far-IR and UV luminosities. First, a well-defined relation exists between the monochromatic luminosity at $1500 \AA$ and the total UV luminosity of a young starburst:

$$
\log L_{\mathrm{UV}}=\log L(1500)+3.2 \pm 0.05 \text {, }
$$

where $L_{\mathrm{UV}}$ is in $\mathrm{ergs}^{-1}$ and $L(1500)$ in $\mathrm{ergs}^{-1} \AA^{-1}$. Eq. (1) simply tells us that the total UV luminosity in a starburst can be obtained from $L(1500)$ after multiplication by 1600 (Leitherer et al. 2002). This observational relation agrees with synthetic models and has its roots in the age independence of the spectrum. $L_{\mathrm{UV}}$ in turn is tightly related to the total bolometric luminosity. STARBURST 99 models predict

$$
\log L_{\mathrm{bol}}=\log L_{\mathrm{uv}}+0.1
$$

where both quantities are in erg s${ }^{-1}$. The UV luminosity accounts for about $75 \%$ of the total luminosity, with a negligible dispersion. The remaining $25 \%$ are mostly in the Lyman continuum, with a small contribution from the Paschen continuum. These relations and their small dispersions are the physical basis for the usefulness of the $1500 \AA$ luminosity to derive star-formation rates from UV continuum fluxes.

An observational verification of this reasoning is in Figure 2, which compares $L_{\text {abs }}$ and $L_{\mathrm{IR}}$ for sample of galaxies observed with HUT and IRAS. $L_{\text {abs }}$ is absorbed UV luminosity, derived observationally after applying the attenuation correction. If eqs. (1) and (2) hold, $L_{\text {abs }}$ should equal $L_{\mathrm{IR}}$ since $L_{\mathrm{abs}} \approx L_{\mathrm{bol}}$. The figure suggests that overall this is the case. The agreement holds over almost three orders of magnitude from low-luminosity galaxies like NGC 4214 to the luminous IR galaxy NGC 6090. The relation between the absorbed and re-emitted flux in the UV and IR, respectively, is generally well defined in UV-selected galaxies (Meurer et al. 1999). On the other hand, it is known to break down in ULIRGs (Goldader et al. 2002). Using the absorbed UV flux would underpredict the far-IR emission by factors of 10-100. Apparently, a simple clumpy foreground screen model for the dust is inappropriate for starburst galaxies with bolometric luminosities in excess of $10^{12} \mathrm{~L}_{\odot}$.

One possible interpretation is that the star-forming regions of ULIRGs are so obscured that we detect no UV light from them at all. In this case, the UV light we see would come from relatively lightly reddened star-forming regions and stars outside the most obscured regions, giving blue colors. High-resolution UV and IR imaging with $H S T$ of a sample luminous and ultra-luminous IR galaxies by Goldader et al. (2002) supports this suggestion. One of the objects, VV 114 $(d=90 \mathrm{Mpc})$ was discovered as a luminous far-IR source in the IRAS Bright Galaxy Survey. VV 114 is a pair of galaxies with a remarkable contrast from the 


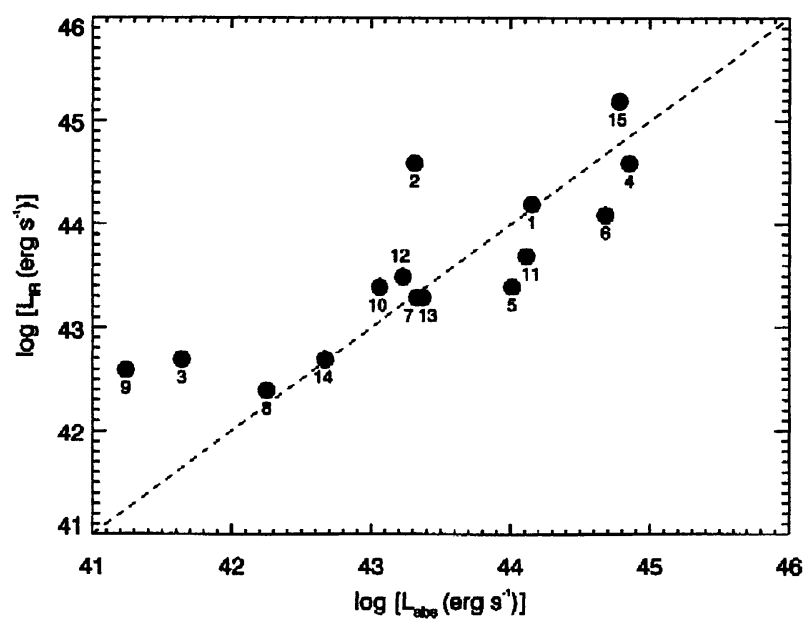

Figure 2. Comparison of $L_{\text {abs }}$, the absorbed UV luminosity between 912 and $3650 \AA$, and the IRAS far-IR luminosity for a sample of star-forming galaxies. Galaxy identifiers: 1: NGC 1097, 2: NGC 1365, 3: NGC 2403, 4: IRAS 08339+6517, 5: NGC 2903, 6: NGC 3310, 7: NGC 3351, 8: NGC 4214, 9: NGC 4449, 10: NGC 5055, 11: Mrk 66, 12: NGC 5194, 13: NGC 5236, 14: NGC 5253, 15: NGC 6090. From Leitherer et al. (2002).

UV to the near-IR. The western galaxy VV 114W has a large number of luminous star-forming regions visible in Figure 3. The brightest UV sources are clearly displaced from the near-IR peaks by at least hundreds of parsecs. Furthermore, only a few percent of the total near-UV light is projected within the inner $500 \mathrm{pc}$ radius, even though this is the region where most of the bolometric energy is generated.

VV 114 highlights the challenge of measuring the rate of star formation at high redshift where morphological information on galaxies is limited and where the stellar content of the most luminous galaxies is often inaccessible to spectroscopic observations. The best studied group of galaxies at cosmological distance is defined by the absence of emission in the $U$-band as a result of the Lyman break at $912 \AA$ (Steidel et al. 1998). They are usually referred to as Lyman break galaxies. Their colors are observed to be redder than expected for dust-free star-forming objects. If due to dust obscuration, the previous line of arguments can be used to predict their far-IR emission, which is redshifted into the sub-mm and becomes observable from the ground. A population of highredshift galaxies has indeed been detected at sub-millimeters (e.g., Chapman et al. 2001) but it is presently unknown whether these galaxies are drawn from a population similar to the Lyman break galaxies, or whether the two methods select entirely different types of object at similar redshifts. Correlating the results from surveys at such widely spaced wavelengths is frustratingly uncertain. If cases like VV 114 are not representative for those galaxies dominating the star formation in the universe, the majority of high-redshift star formation occurs in objects which can at least be detected in existing UV-selected surveys, such 

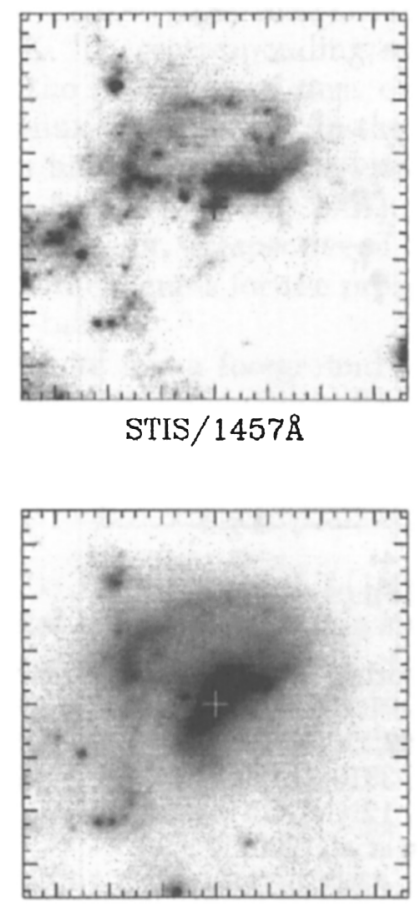

NICMOS $/ 1.1 \mu \mathrm{m}$

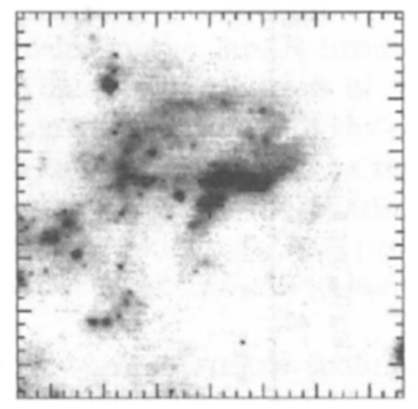

STIS $/ 2364 \AA$

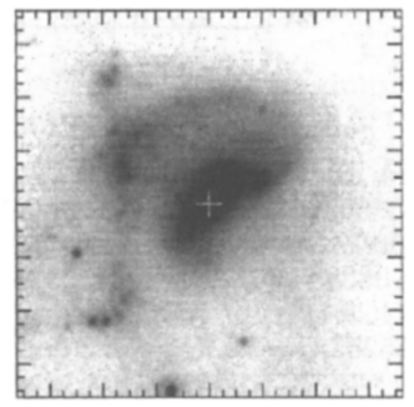

NICMOS $/ 1.6 \mu \mathrm{m}$ VV $114 \mathrm{~W} 3 \times 3 \mathrm{kpc}$

Figure 3. Inner $3 \mathrm{kpc}$ of VV $114 \mathrm{~W}$ imaged with HST-STIS (upper) and NICMOs (lower). The maximum brightness in the IR is marked with a cross. From Goldader et al. (2002).

as Lyman break galaxies (Adelberger \& Steidel 2000). Sub-mm galaxies would then be the dust-rich tail of the star-forming galaxy population.

Acknowledgments. Scott Chapman provided access to a Keck spectrum of a JCMT-SCUBA source prior to publication. Torsten Böker's comments helped improve an earlier version of the manuscript. STScI is operated by AURA, Inc., for NASA under contract NAS5-26555.

\section{References}

Adelberger, K.L., Steidel, C.C. 2000, ApJ 544, 218

Böker, T., van der Marel, R.P., Vacca, W.D. 1999, AJ 118, 831

Bresolin, F., Kennicutt, R.C. 2002, ApJ 572, 838

Calzetti, D. 2001, PASP 113, 1449

Chapman, S.C., Richards, E.A., Lewis, G.F., et al. 2001, ApJ (Letters) 548, L147

Cid Fernandes, R., Heckman, T.M., Schmitt, H., et al. 2001, ApJ 558, 81

Doherty, R.M., Puxley, P., Doyon, R., Brand, P.W.J.L. 1994, MNRAS 266, 497

Genzel, R., Lutz, D., Strurm, E., et al. 1998, ApJ 498, 579

Genzel, R., Cesarsky, C.J. 2000, Ann. Review Astron. Astrophys. 38, 761 
Gilbert, A.M., Graham, J.R. 2002, in: D. Geisler, E.K. Grebel \& D. Minniti (eds.) Extragalactic Star Clusters, Proc. IAU Symp. No. 207 (San Francisco: ASP), p. 471

Goldader, J.D., Meurer, G., Heckman, T.M., et al. 2002, ApJ 568, 651

González Delgado, R.M., Leitherer, C., Stasińska, G., et al. 2002, ApJ 580, 824

Heckman, T.M., Armus, L., Miley, G.K. 1990, ApJS 74, 833

Ho, L.C., Filippenko, A.V. 1996a, ApJ (Letters) 466, L83

,$- 1996 \mathrm{~b}$, ApJ 472, 600

Leitherer, C., Vacca, W.D., Conti, P.S., et al. 1996, ApJ 465, 717

Leitherer, C., Schaerer, D., Goldader, J.D., et al. 1999, ApJS 123, 3

Leitherer, C., Li, I.-H., Calzetti, D., Heckman, T.M. 2002, ApJS 140, 303

Lutz, D., Kunze, D., Spoon, H.W.W., Thornley, M.D. 1998, A\&A (Letters) 333, L75

Maoz, D., Ho, L.C., Sternberg, A. 2001, ApJ (Letters) 554, L139

Massey, P., Hunter, D.A. 1998, ApJ 493, 180

Meurer, G.R., Heckman, T.M., Calzetti, D. 1999, ApJ 521, 64

Oliva, E., Origlia, L., Kotilainen, J.K., Moorwood, A.F.M. 1995, A\&A 301, 55

Rieke, G.H., Loken, K., Rieke, M.J., Tamblyn, P. 1993, ApJ 412, 99

Schaerer, D., Vacca, W.D. 1998, ApJ 497, 618

Schaerer, D., Contini, T., Pindao, M. 1999, A\&AS 136, 35

Schaerer, D., Stasińska, G. 1999, A\&A 345, 17

Schmutz, W., Leitherer, C., Gruenwald, R. 1992, PASP 104, 1164

Smith, L.J., Gallagher, J.S. 2001, MNRAS , 326, 1027

Smith, L.J., Norris, R.P.F., Crowther, P.A. 2002, MNRAS 337, 1309

Steidel, C.C., Adelberger, K.L., Dickinson, M., et al. 1998, ApJ 492, 428

Thornley, M.D., Förster Schreiber, N.M., Lutz, D., et al. 2000, ApJ 539, 641

Vacca, W.D., Conti, P.S. 1992, ApJ 401, 543

Veilleux, S. 2001, in: L. Tacconi \& D. Lutz (eds.), Starburst Galaxies: Near and Far, (Berlin: Springer), p. 88

Viegas, S.M., Contini, M., Contini, T. 1999, A\&A 347, 112

\section{Discussion}

WALBORN: Giant Hil regions are probably not relevant to monolithic starbursts on much larger scales, and they will not elucidate global triggering and propagation. However, if the starburst breaks up into substructure on $\mathrm{H}$ II-region scales (as in, e.g., HST images of NGC 4214 and the Antennae galaxies NGC 4038/9), then the structure and evolution of highly resolved nearby objects is very relevant. Moreover, 30 Doradus contains stellar substructures of four different ages, including a triggered IR generation and an extraneous $20 \mathrm{Myr}$-old cluster with red supergiants. Could all of that be extracted reliably from the integrated spectrum of a distant, unresolved object? I think it is a good idea to be guided by the resolved information from local objects.

LEITHERER: IR bright and luminous galaxies are likely to be closer to the quasimonolithic case than being similar to a single 30 Dor. One indication is the effect of the starburst on the ISM of the host galaxy. In most cases, a large-scale galactic superwind is initiated by the starburst, disrupting the ISM and governing the galaxy evolution. This is certainly not the case with a single $\mathrm{H}$ II region in a dwarf galaxy. It is indeed important to study the microphysics of star-forming regions like 30 Dor. Fortunately, it appears that its global properties can be derived even from less detailed observations. 
Vacca et al. (1995) interpreted large-aperture optical and UV data of the central $\sim 1^{\prime}$. The integrated spectra were used to predict the stellar number counts of O-type and WR stars. The outcome was quite satisfactory.

ZINNECKER: What do you expect to be the most important breakthroughs from NGST and ALMA?

LEITHERER: I personally am excited about the prospects of very deep and highly resolved sub-mm ovservations of distant star forming galaxies and of rest frame-UV spectroscopy of these galaxies at $z<5$. This will allow us to perform studies surpassing the quality of those we are now doing at $z \lesssim 3$.

CERviÑo: In your talk you have mentioned that with RSG it is possible to quote the mass into stars from star-forming regions. However, any determination of starburst properties based in post-main-sequence stars (WR, RSG, AGB, etc.) may be strongly affected by the discretness of the stellar population and the sampling of the IMF (at least more affected than main-sequence stars ). So it is needed to take these sampling effects into account for safe comparisons. It is not the case in some of your examples (clusters mass function, or the case of high metalllicity $\mathrm{H}$ II regions). As a safety limit about some numbers of RSG (or WR) are needed for realistic comparison of synthesis models and real data.

LEITHERER: I agree with you in that small number statistics can be a serious issue for low-luminosity starbursts and $\mathrm{H}$ II regions. In a way, you are giving precisely the opposite advice as Nolan Walborn: observe large, distant starbursts to improve the number statistics. And these are the objects I was mostly talking about. To put this into perspective: even an average starburst like M 82 has many thousands of red supergiants in an observed spectrum.

VACCA: A word of caution regarding the interpretation of the $F_{\mathrm{FIR}} / F_{\mathrm{UV}} v s . \beta$ correlation. We know of two examples in the local universe (He2-10 and NGC 5253) in which the distribution of the FIR flux is not at all correlated with the distribution of the UV flux, even though these objects obey the $F_{\mathrm{FIR}} / F_{\mathrm{UV}} v s . \beta$ correlation. Hence these objects show that the interpretation of this correlation as the result of dust re-processing UV flux into FIR in a foreground screen cannot be correct for all objects. In some sources the FIR flux is produced by regions that are so embedded that they can never be seen in the UV.

LEITHERER: One should consider the $F_{\mathrm{FIR}} / F_{\mathrm{UV}} v s . \beta$ plot an average relation, which applies to UV selected galaxies in the local universe. Most - but not all - galaxies follow the relation. There are galaxies which are outliers, like NGC 5253 or He2-10, but then there are many more galaxies which fit very well. I would take the relation as a useful recipe, which cannot be derived from first principles, but gives, on average, the right answer.

CROWTHER: I agree that we should be very wary about over-interpreting average IR fine-structure ratios (e.g., [Ne III]/[NeII]) in starburst galaxies, where we can resolve these lines, such as NGC 5253. We see the regions of [Ne III] formation, containing very massive hot $\mathrm{O}$-type stars, distinct from regions with [Ne II] emission, containing an older population.

LEITHERER: You are right, NGC5253 is a case where the small-scale structure is obvious and where it matters. This argument, of course, applies to observations at all wavelengths (including the visual) and to objects which do not show a soft radiation field in their emission-line spectra. 